\title{
Solving nonlinear wave equation based on topology
}

\author{
Liang Song*, Guihua Li, Shaodong Chen \\ School of Mathematics and Physics, Nanyang Institute of Technology, \\ Nanyang, 473004 \\ China
}

Received: March 1, 2021. Revised: July 30, 2021. Accepted: August 28, 2021. Published: August 31, 2021.

\begin{abstract}
A method of solving nonlinear wave equation based on topology is proposed. Firstly, the characteristics of stochastic graph and Scaleless network are compared, and their topological characteristics are analyzed. Because of the existence of a few axis nodes, Scaleless networks have higher average aggregation than those with the same number of airport nodes and connected stochastic graphs. According to the topological structure of nonlinear wave equation, the first-order integral method is used to solve the nonlinear wave equation. According to the first integration, the threshold range is set, and the solution flow is designed in line with the division theorem. The topology of the network is analyzed according to the node degree, aggregation coefficient and reciprocity of the network, so as to verify and analyze. The experimental results show that the application of this method is $98 \%$, which is still effective for the hyperbolic development equation of the same type.
\end{abstract}

Keywords-Nonlinear, solution, topology, wave equation.

\section{INTRODUCTION}

$\mathrm{T}$ HE existence and multiplicity of solutions to PDEs (Partial Differential Equation) is an important field in modern science and an important part of the theory of PDEs. As a branch, the multiplicity of solutions of nonlinear wave equation and bridge model equation not only has important theoretical value, but also has important practical significance for mechanics, physics, engineering and other disciplines [1]. Some projects, such as bridges and dams, can establish wave equations. The study of the multiplicity of solutions of nonlinear wave equations provides necessary theoretical support for these practical problems and ensures the smooth progress of the project. The variational method in differential equation is to turn the boundary value problem of differential equation into the variational problem to certify the entity, number and approximate solution of the solution. To be specific, many problems in natural science and engineering technology often require such an abstract function functional, whose value domain is the real number domain, and the definition domain is composed of some function [2]. It is the basic problem of variational method to study the extremum of functional. As a branch of mathematical analysis, variational method is an extension of the method of dealing with extreme value of function in differential calculus, but the function in the domain of functional definition plays the role of independent variable. When dealing with extremum problems, the variables suitable for functional extremum conditions are not a single or finite number of numerical variables, but the whole changing curve or function, or even a group of functions, so it involves more in-depth and extensive problems [3]-[5].

Nonlinear partial differential equations (PDEs) are widely used in the fields of hydrodynamics, plasma physics, optics, solid-state physics and transportation. Due to the complexity of the problem and the influence of nonlinear terms, it is almost impossible to solve the analytical solution, so it is of great significance to solve the exact traveling wave form of non-linear partial differential equation (Group) [6]. Network is an objective phenomenon in nature and social system, which follows certain laws of nature and social economy. In abstract terms, a network can represent edges between many nodes and connecting nodes, where the nodes are used to represent the different elements in the real system and the edges are used to represent the relationships between individuals. Usually, when two nodes have a relationship, they connect to an edge; otherwise, they do not. Two nodes connected by an edge are considered adjacent nodes in the network [7]. In this sense, the network can be seen as a network of nodes connected with each other through edges. The properties that the network can display without depending on the specific location of the node and the specific shape of the edge are called the topological features of the network, and the corresponding structures are called the 
network topology features [8]. With the development of solitary wave theory, some methods have been reported to solve the exact settles of nonlinear partial differential equations, like the inverse scattering transform, hyperbolic tangent hyperbolic secant method, homogeneous balance method, exponential function method, expansion method and first integration method. Reference [9] proved that Ball Marsden Slemrod controllability barrier is also valid for nonlinear equations with linear control. Firstly, an abstract result is given, and then it is applied to nonlinear wave equations. The first application of sine Gordon equation comes directly from abstract results, and the second application involves cubic wave / Klein Gordon equation. Reference [10] considers the surface wave propagating along the dielectric interface with third-order nonlinear polarizability and topological insulator. The optical nonlinearity of the medium ensures the existence of surface waves. For dielectrics with positive or negative linear permittivity, the spin angular momentum density of surface waves has been determined. The results show that the discreteness of topological number is represented by the discreteness of normal component and tangential component of spin angular momentum density. At the dielectric interface, the increase of the electric field intensity of the wave will change the value of the spin angular momentum and may lead to its disappearance.

In this paper, the first integration method is used to solve the exact traveling wave solution of nonlinear wave equation. The advantage of the first integration method is that the whole iterative algorithm is pure algebraic operation, so it can be solved with the help of symbolic calculation software. Based on this, research on solving nonlinear wave equation based on topology structure is proposed. According to the characteristics of topological structure, the first integration method is used to solve the exact solution of nonlinear wave equation. Through the empirical analysis of this method, the application effect of this method in solving nonlinear wave equation is improved. The effectiveness of the method is verified.

\section{MATERIALS AND METHODS}

There are three kinds of network topology, conventional network, stochastic graph and complex network (mainly scaleless network). The geometric parameters of all nodes in the regular network are the same, which does not conform to the actual state of the real network. Most networks are dynamic evolution open networks, which are the result of the interaction of randomness and regularity. Therefore, the characteristics of stochastic graph and Scaleless network can be compared and analyzed. On this basis, the reasonable topological structure of network can be preliminarily defined. The Scaleless network has a higher average aggregation degree than the stochastic graph with the same number of airport nodes and connections. The index is used to evaluate the network connectivity and loop. According to topology features, the first integration method is used to solve the exact solution of nonlinear wave equation. Set the threshold range according to the first integration, and design the solution flow according to the division theorem.

\section{A. Topology}

The network in each system has its own special nature and formation mechanism. For the traffic network, each node wants to establish direct, fast and convenient connection with other nodes, but it is often limited by various constraints and cannot form a complete connection state. Although there is no objective restriction condition in the ground transportation network, any node of the network can establish direct linear contact with other nodes. Economic calculation and government regulation are the factors that restrict the existence of boundary between the two nodes. Government regulation mainly refers to the examination and approval constraints of opening, while economic calculation refers to the consideration of operating profit of enterprises. When the market demand between the two nodes reaches a certain threshold of passenger flow, that is, the revenue is greater than the cost, the company may realize the connection of the side.

According to this situation, the network characteristics of stochastic graph and Scaleless network are compared, as shown in Table I.

Table I. Contrast between stochastic graph and Scaleless network

\begin{tabular}{ccc}
\hline Contrast parameter & stochastic graph & Scaleless network \\
\hline $\begin{array}{c}\text { Node degree } \\
\text { division }\end{array}$ & Poisson Division & Power-law Division \\
$\begin{array}{c}\text { Featureless scale } \\
\text { Network average } \\
\text { distance }\end{array}$ & Mean value & Non-characteristic scale \\
$\begin{array}{c}\text { Network cluster } \\
\text { coefficient }\end{array}$ & Small & Small \\
Axis node & Small & Big \\
\hline
\end{tabular}

For the network, the most directly related two important parameters that reflect the topological characteristics of the network are the average distance and cluster coefficient of the network. In the network, the average distance represents the depth of transportation, and the cluster coefficient represents the breadth of transportation. The characteristics of fast and convenient transportation must require a small depth of transportation, that is, two points in the network can be reached through as few connections as possible [10] [11]. In the transportation network, the cluster coefficient represents the average aggregation degree of the network composed of the general field and the adjacent airport nodes, and represents the completeness index of the network [12].

Due to the existence of a few axis nodes, the Scaleless network has a higher average aggregation degree than the stochastic graph with the same number of airport nodes and connections. Airport nodes are more closely related to each other and the network is more complete. In other words, due to the existence of axis nodes, stochastic graphs have a larger average distance than Scaleless networks with the same degree of aggregation. To reduce the average distance to meet the requirements of transportation, it is necessary to increase the average number of connections (increase the number of lines), 
which will lead to the decline of network operation efficiency. Therefore, for real networks, Scaleless networks have better completeness and higher network efficiency than stochastic graphs in terms of network characteristics [13]. In order to express the structure of the network more clearly, we can use $\alpha$ index, $\beta$ index and $\chi$ index to evaluate the connectivity and loop of the network [14].

\section{B. Solution of Nonlinear Wave Equation}

According to topology features, the first integration method is used to solve the exact solution of nonlinear wave equation.

As:

$\mathrm{a}_{t t}+\alpha \mathrm{a}_{x x}+\beta a+\chi a^{3}=0$

where $\alpha>0, \beta, \chi$ are real constants. (1) can be transformed into ordinary differential equation by $\mathrm{a}(x, t)=g(\xi), \xi=x-\eta t$ :

$\eta^{2} \mathrm{~g}^{\prime \prime}(\xi)+\alpha \mathrm{g}^{\prime \prime}(\xi)+\beta \mathrm{g}(\xi)+\chi \mathrm{g}^{3}(\xi)=0$

According to the first integration, it is assumed that $\mathrm{g}(\xi)$ is a nontrivial solution of the formula, and it is an irreducible polynomial within the threshold range. According to the Division theorem, there is a polynomial that satisfies the following equation within the threshold range.

$\frac{\mathrm{d} q}{d \xi}=\frac{\vartheta q}{\vartheta X} \frac{\vartheta X}{\vartheta \xi}+\frac{\vartheta q}{\vartheta Y} \frac{\vartheta Y}{\vartheta \xi}=(g(X)+h(X) y) \sum_{i=0}^{m} a_{i}(X) y^{i}$

If the coefficients on both sides of the equation are the same, the polynomial can be obtained [15]. The first integration method is a feasible and effective the method of finding the exact solution of this nonlinear wave equation. Therefore, it can also be used to find the exact solution of similar hyperbolic partial differential equations such as nonlinear beam equation and nonlinear Schrodinger equation.

The solution flow of the design is shown in Fig. 1.

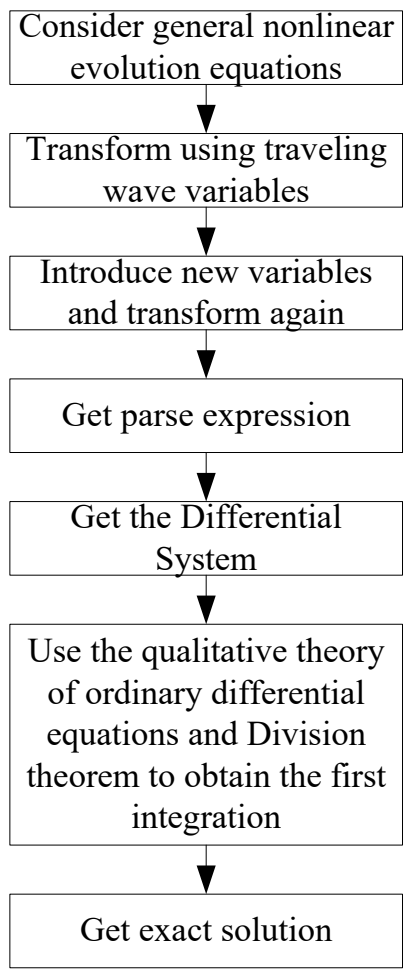

Fig. 1 solution flow

The specific solution process is as follows:

The basic idea of the first integral method is to transform nonlinear partial differential equations into ordinary differential equations by traveling wave transformation. The ordinary differential equations are appropriately changed to be converted into ordinary differential equations. Finally, the exact solution of the nonlinear partial differential equation is solved. Proceed as follows:

1) General nonlinear development equation:

$T\left(h, h_{t}, h_{x}, h_{t t}, h_{x t}, h_{x x x}\right)=0$

2) The traveling wave variable $\xi=x-c t$ is used to perform $\mathrm{h}(x, t)=U(\xi)$ transformation on (4) to obtain:

$T\left(U, U^{\prime}, U^{\prime \prime}, U^{\prime \prime \prime}\right)=0$

3) Assuming a new variable $h(x, t)=f(\xi)$ is introduced, then (5) can be transformed into:

$X(\xi)=f(\xi), Y=f_{\xi}(\xi)$

where the $\mathrm{f}$ is the analytical expression that can be obtained from (5), so as to obtain a differential system:

$X_{\xi}(\xi)=Y(\xi), Y_{\xi}=F(X(\xi), Y(\xi))$

4) The $F$ is the derivative expression about $Y$ obtained from (7). First using the qualitative theory of ordinary differential equations and Division theorem, a first integration of (4) is obtained, and then substituted into (6) to obtain the solution of the equation, that is, the exact solution of (4) is obtained.

\section{RESULTS}

For verification the rationality of research on solving nonlinear wave equation based on topology structure, an 
empirical analysis is performed.

\section{A. Sample Data}

The banks under construction include 3 national policy banks, 4 state-owned banks and 12 joint-stock banks. Other city commercial banks, urban credit cooperatives, rural credit cooperatives and postal savings institutions can participate in the interbank lending market, but the lending amount in the interbank lending market is small or data collection is difficult, so the banking network studied in this paper does not include these financial institutions. For the convenience of research, the banks studied are numbered: 1 is Industrial and Commercial Bank of China; The second is the Agricultural Bank of China; The third is the Bank of China; 4. China Construction Bank; Fifth, Bank of Communications; 6 is ZTE Bank; 7 is Hua Xia Bank; 8 is China Minsheng Bank; 9 is Guangdong Development Bank; 10 is Shenzhen Development Bank; 11 is China Merchants Bank; 12 is Industrial Bank; 13 is Shanghai Pudong Development Bank; 14 is hengfeng bank; 15 is Zhejiang Commercial Bank; China Everbright Bank 16; 17 people from China Development Bank; 18 is the Exim Bank of China; The 19th is the Agricultural Development Bank of China.

The data studied in this article are from the 2009 balance sheets of 19 banks in the 2010 China Financial Yearbook. The total inter-bank assets are the sum of interbank deposits and inter-bank placements in the balance sheet, and the inter-bank liabilities are the sum of inter-bank deposits and inter-bank placements in the balance sheet (unit: 100 million yuan). In this paper, the above matrix method is used to calculate the interbank borrowing scale of 19 banks. The ratio of interbank assets (liabilities) to all interbank assets (liabilities) can be obtained. The data processing and analysis of the full text is realized through software such as Matlah, Ucinet. MATLAB is a commercial mathematical software produced by MathWorks company, which is used in data analysis, wireless communication, deep learning, image processing and computer vision, signal processing, quantitative finance and risk management, robot, control system and other fields. UCINET network analysis integration software includes Netdraw for one-dimensional and two-dimensional data analysis, mage for three-dimensional display analysis, and pajek free for large-scale network analysis. Using UCINET software can read text files, krackplot, pajek, negopy, VNA and other formats. It can handle 32767 network nodes.

\section{B. Network Construction}

Each institution shall be numbered in the order of state-owned banks, joint-stock banks, urban commercial banks, rural commercial banks, foreign-funded banks, policy banks, postal savings banks and non-bank financial institutions. The naming method is "No. + number", "balance" is No. 99, and the time span is 2013-2018. With banks and other financial institutions as nodes, edges are established based on the estimated interbank relationship matrix. In the end, an interbank network in China was formed, as shown in Fig. 2.

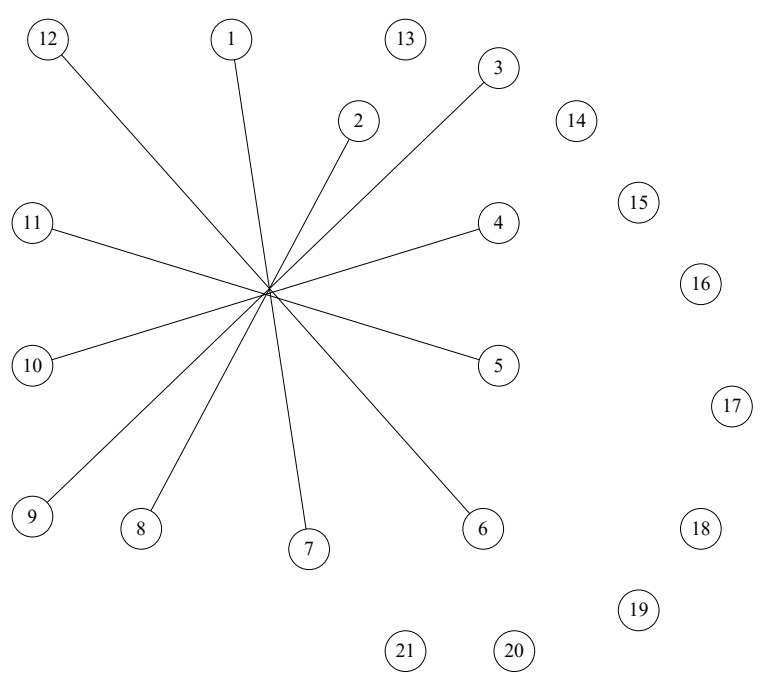

(a) 2013

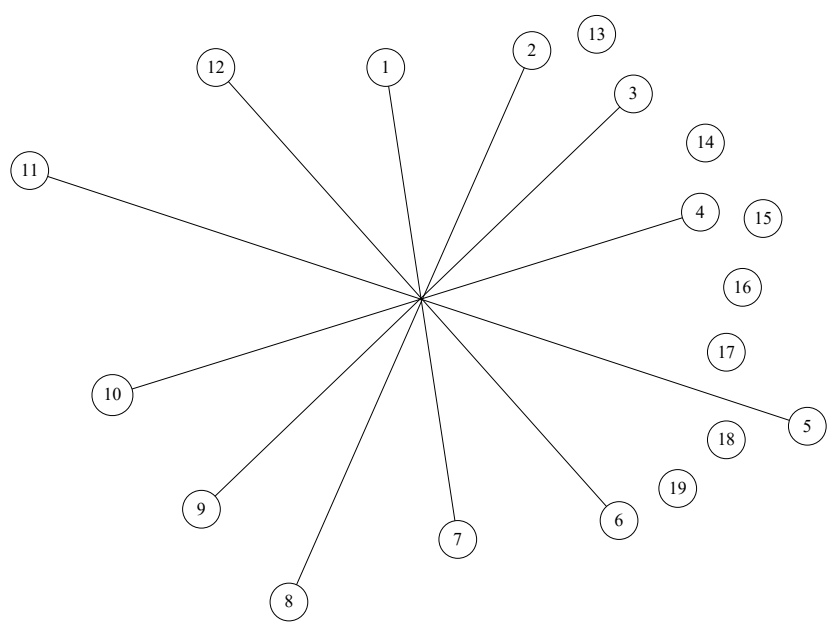

(b) 2014

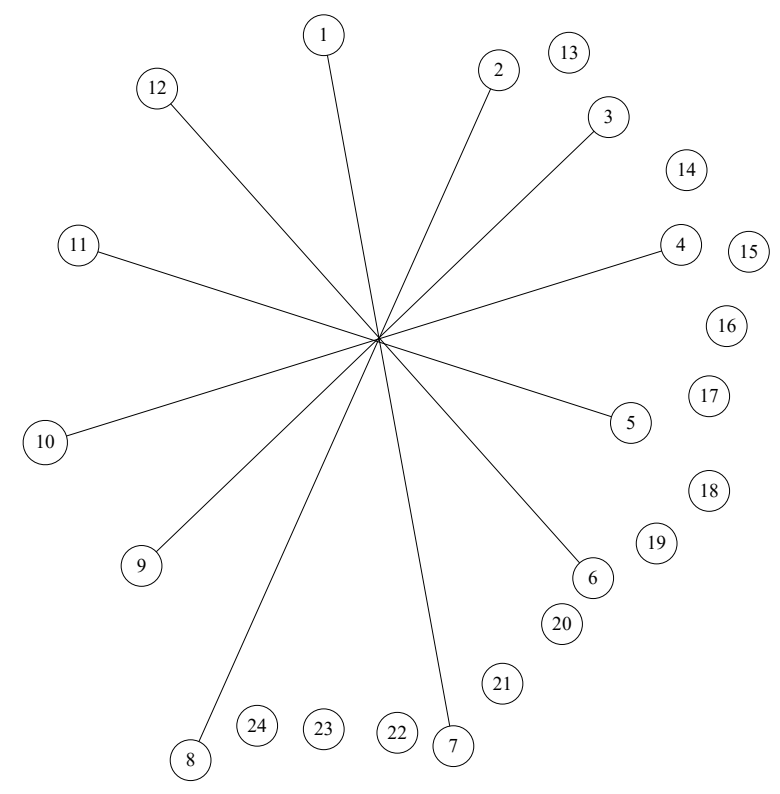

(c) 2015 


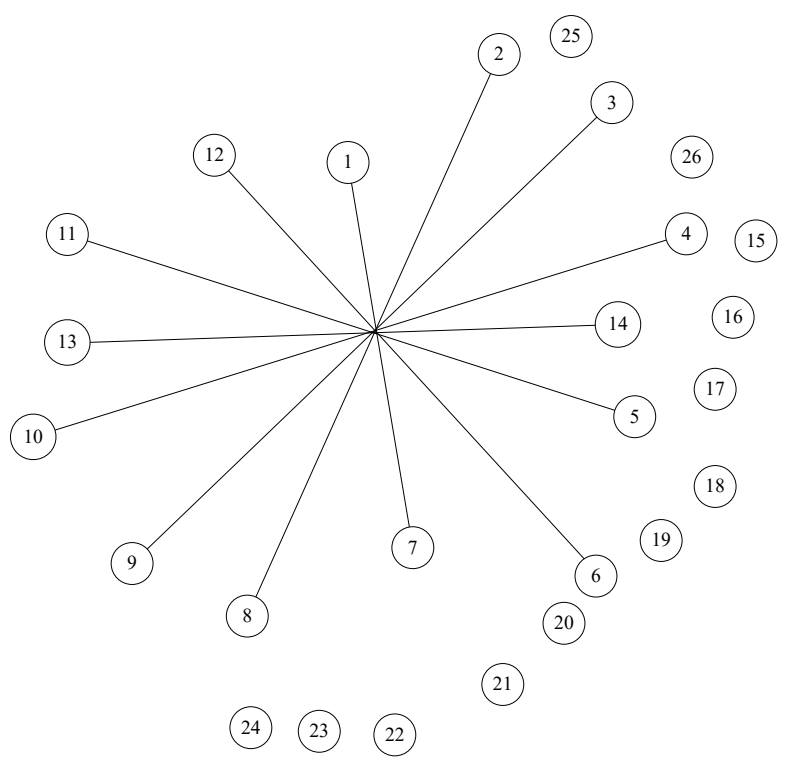

(d) 2016

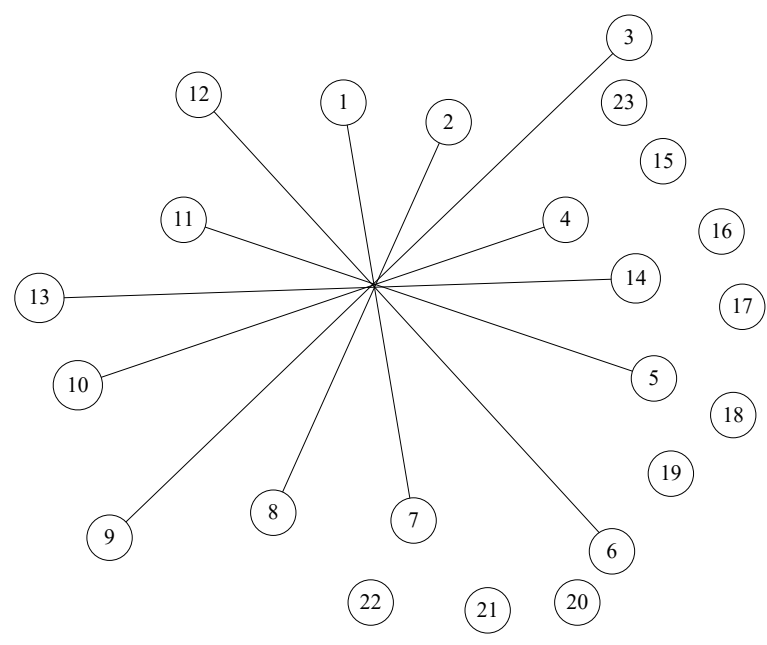

(e) 2017

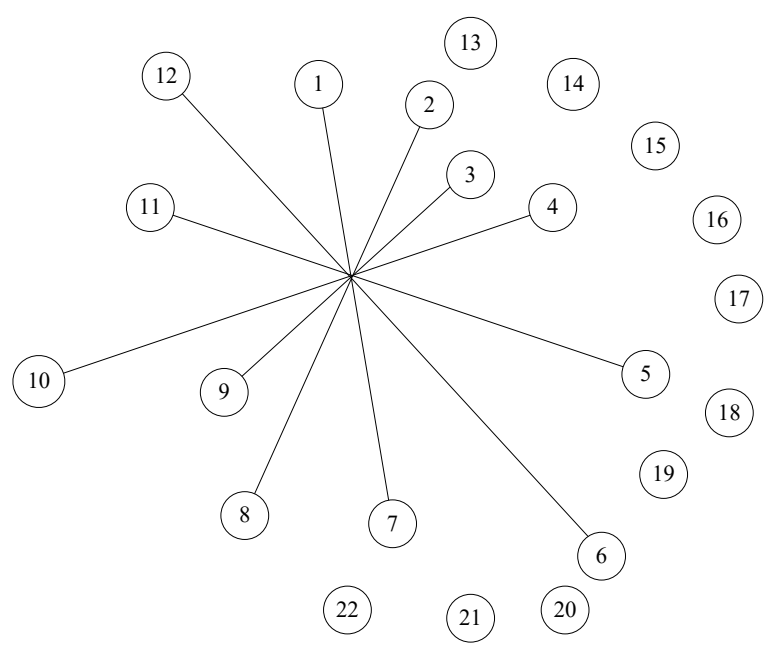

(f) 2018

Fig. 2 inter-bank network from 2013 to 2018
The data in the figure represents the inter-bank network node. Different banks represent different network nodes. Because the market structure obtained by the maximum moisture method is a complete structure, that is, there are transactions between any two inter-bank, which is obviously inconsistent with the reality, but the scale is different, so the side with smaller scale of inter-bank transactions is eliminated. Due to the two-way transaction between banks, interbank assets and liabilities will be generated at the same time, so the constructed network is a directed network. As can be seen from Fig. 2, the structure of China's interbank network is relatively stable from 2013 to 2018, showing heterogeneous and complex structural characteristics. Some highly connected nodes act as "hubs", mainly large commercial banks. In addition, there are some independent nodes that are not connected with other nodes, mainly urban commercial banks and agricultural commercial banks.

\section{Network Topology}

(1) Node degree. The degree of a node represents the number of edges connected to that node in the network. Each node of the directed bank network has an out degree and an in-degree. The out-degree (I) of bank a represents the number of debt banks that the bank a has, and the in-degree (i) of bank a represents the number of creditor banks that the bank a has. If the bank a has a high out-degree (in-degree), it means that bank a has a lot of inter-bank liabilities (risk exposure). The corresponding calculation formula of in degree and out degree is as follows:

in $-\operatorname{deg} \operatorname{ree}(i)=\sum_{j=1}^{N} e_{j i}$, out $-\operatorname{deg} r e e(i)=\sum_{j=1}^{N} e_{j i}$

The out-degree and in-degree of the bank network nodes under different thresholds are shown in Table II. 
Table II. The out-degree and in-degree of the bank network nodes under different thresholds

\begin{tabular}{|c|c|c|c|c|c|c|}
\hline \multirow{2}{*}{ Nodes } & \multicolumn{2}{|c|}{$\mathrm{C}=9 \times 10^{-4}$} & \multicolumn{2}{|c|}{$\mathrm{C}=0.002$} & \multicolumn{2}{|c|}{$\mathrm{C}=0.004$} \\
\hline & out-degree & in-degree & out-degree & in-degree & out-degree & in-degree \\
\hline 1 & 14 & 16 & 11 & 14 & 6 & 9 \\
\hline 2 & 13 & 14 & 8 & 9 & 6 & 6 \\
\hline 3 & 14 & 14 & 13 & 12 & 11 & 6 \\
\hline 4 & 14 & 15 & 9 & 12 & 6 & 7 \\
\hline 5 & 13 & 14 & 8 & 9 & 6 & 6 \\
\hline 6 & 9 & 10 & 6 & 5 & 1 & 1 \\
\hline 7 & 6 & 6 & 1 & 2 & 0 & 1 \\
\hline 8 & 9 & 10 & 6 & 5 & 0 & 1 \\
\hline 9 & 7 & 5 & 3 & 1 & 0 & 0 \\
\hline 10 & 2 & 3 & 0 & 0 & 0 & 0 \\
\hline 11 & 11 & 14 & 6 & 9 & 6 & 5 \\
\hline 12 & 10 & 12 & 6 & 6 & 5 & 6 \\
\hline 13 & 9 & 7 & 3 & 2 & 1 & 1 \\
\hline 14 & 0 & 0 & 0 & 0 & 0 & 0 \\
\hline 15 & 1 & 0 & 0 & 0 & 0 & 0 \\
\hline
\end{tabular}

Table II shows the in-degree and out-degree of bank network nodes calculated under the thresholds of $9 \times 10^{-4}, 0.002$ and 0.004 respectively. Through the calculation results under different thresholds, it can be seen that the nodes that keep relatively high outgoing and incoming degrees are 1, 2, 3, 4, 5. That is to say, in the interbank lending market, industrial and Commercial Bank of China, Agricultural Bank of China, Bank of China, China Construction Bank and Bank of communications have a relatively high frequency of interbank lending with other banks, and have a relatively active performance. They are in the core position in China's interbank lending market, which just reflects that these five banks have strong capital and high credit level in China's banking system. However, from the perspective of the out-degree and in-degree of nodes 14 and 15, Hengfeng bank and Zheshang Bank hardly carry out credit lending between banks.

(2) Aggregation factor. The aggregation coefficient of network is an important parameter to measure the degree of network clustering. It is a local characteristic quantity, which refers to the probability that two nodes directly connected to a given node happen to be also directly connected to each other. For node $\mathrm{i}$ with $\mathrm{K}_{\mathrm{i}}$ edges, the aggregation coefficient $\mathrm{Ci}$ is defined as:

$C_{\mathrm{i}}=2 n_{i} / k_{i}\left(k_{i}-1\right)$

where the $n_{i}$ represents the number of edges between the $k_{i}$ neighbors of node I. generally, the directionality of edges is ignored in the calculation of aggregation coefficient for directed networks. If $\mathrm{C}_{\mathrm{i}}=0$, no neighbors of node $\mathrm{i}$ are connected; If $\mathrm{C}_{\mathrm{i}}=$ 1 , all neighbors of node $\mathrm{i}$ are connected. The high aggregation shows that there is good connectivity between the neighbors around the node. The average clustering coefficient $<\mathrm{C}>$ of the network is the average value of the clustering coefficient of all the vertices in the network. The average clustering coefficient $<$ $\mathrm{C}>$ of the network describes the average clustering degree of the whole network.

The average aggregation coefficient of the bank network at different thresholds is calculated (see Fig. 3).

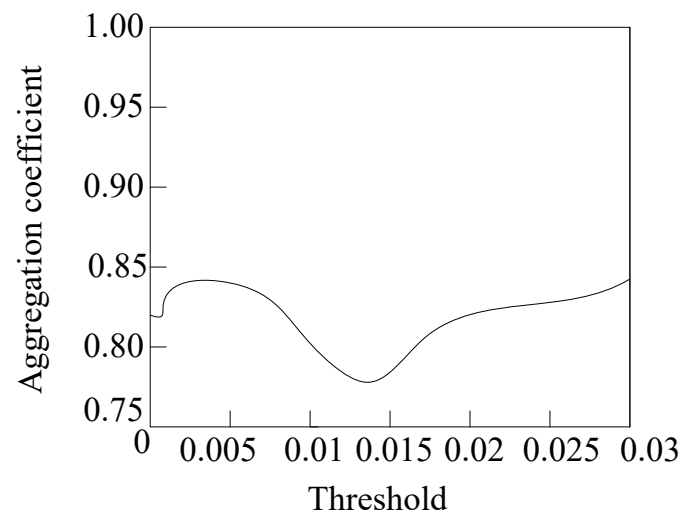

Fig. 3 average aggregation coefficient of the bank network under different thresholds

Although the density of bank network edge decreases with the increase of threshold, the average aggregation coefficient of bank network does not decrease with the increase of threshold. Sometimes, under a high threshold, the banking network has a high average aggregation coefficient. For example, when $\mathrm{C}=0.030$, the average aggregation coefficient of the banking network is 0.833 . On the whole, the banking network has a relatively large average aggregation coefficient, which indicates that the average aggregation degree of the banking network in China is relatively high, that is to say, the internal network connection of the adjacent banks in the interbank market is relatively complete. In addition, under different thresholds, the aggregation coefficients of nodes 1,2,3,4,5 are relatively high, especially when the threshold selection is large. This shows that industrial and Commercial Bank of China, Agricultural Bank of 
China, Bank of China, China Construction Bank and Bank of communications have a high degree of network connection with other banks.

(3) Reciprocity. Reciprocity of directed network refers to the problem of two-way edge between nodes in directed network. In the banking network, not all inter-bank lending relationships are two-way, that is, bank $i$ is the creditor bank of bank $j$ does not mean that bank $j$ is the creditor bank of banki. The reciprocity of banking network, that is, the degree of two-way inter-bank lending relationship between two banks in the banking network, is an important measure of the banking network. Because the reciprocity of the banking network has an important impact on the mechanism and speed of risk contagion between banks, the existence of two-way lending relationship will accelerate the process of risk contagion between banks and improve the probability of risk from the initial bank to the target bank. For the reciprocity of the banking network, we use the value of network reciprocity to define:

$$
\lambda=\frac{\sum_{\mathrm{i} \neq \mathrm{j}}\left(\mathrm{e}_{\mathrm{ij}}-\bar{e}\right)\left(\mathrm{e}_{\mathrm{ij}}-\bar{e}\right)}{\sum_{\mathrm{i} \neq \mathrm{j}}\left(\mathrm{e}_{\mathrm{ij}}-\bar{e}\right)^{2}}
$$

According to the value of network reciprocity $\lambda$, networks can be divided into reciprocal networks $(\lambda>0)$ and anti-reciprocal networks $(\lambda<0)$.

For the reciprocity of the banking network, we can find that when the threshold $\mathrm{c}$ is between 0 and $7.390 \times 10^{-8}$ or $7.790 \times$ $10^{-2}$ and 0.091 , the banking network is an anti reciprocal network; When the threshold $\mathrm{c}$ is between $7.400 \times 10^{-8}$ and 0.078 , the banking network is a reciprocal network. The reciprocity coefficient of banking network under different thresholds is shown in Fig. 4.

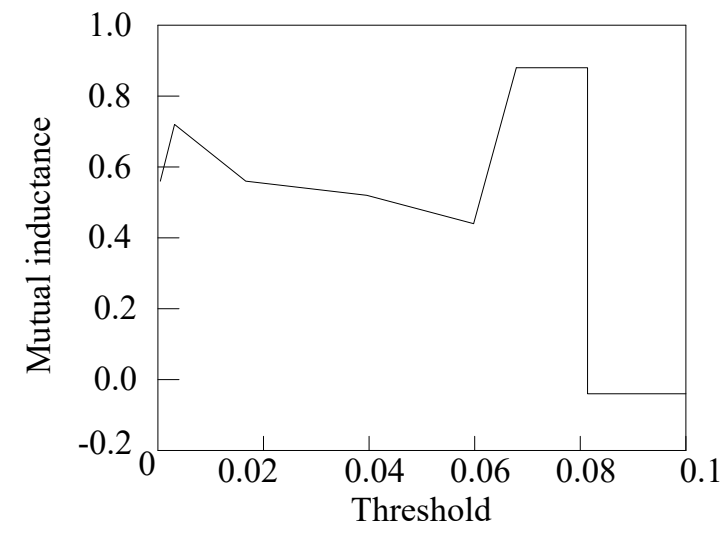

Fig. 4 reciprocity coefficients of banking networks at different thresholds

It can be seen that China's banking network is basically a reciprocal network. In particular, when the $\mathrm{c}$ is between 0.070 and 0.078 , the reciprocity coefficient $\lambda=1$, which means that the inter-bank lending relationship between banks at this time is two-way.

\section{Experimental Results and Analysis}

According to the analysis of the network topology described above, it is known that a comparative analysis needs to be made between traditional application effects and application effects based on the characteristics of the topology structure. The analysis of the characteristics of the four topological structures is shown in Fig. 5 (The curve in the figure is affected by interference).

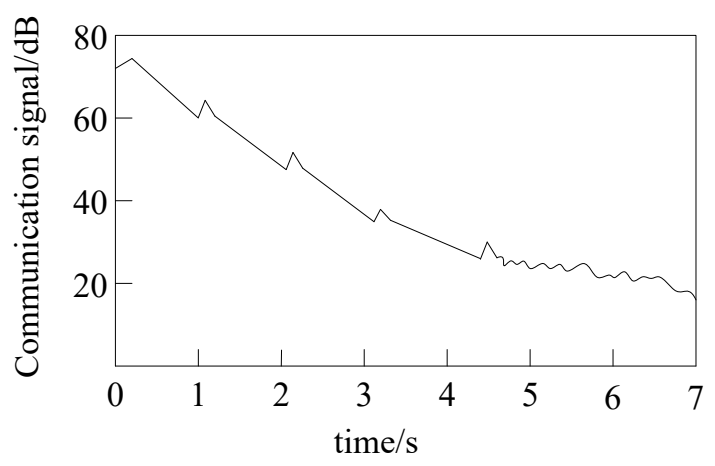

(a) topology feature 1

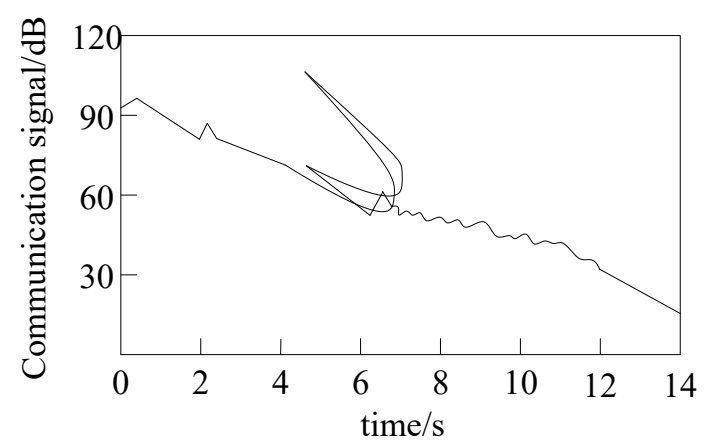

(b) topology feature 2

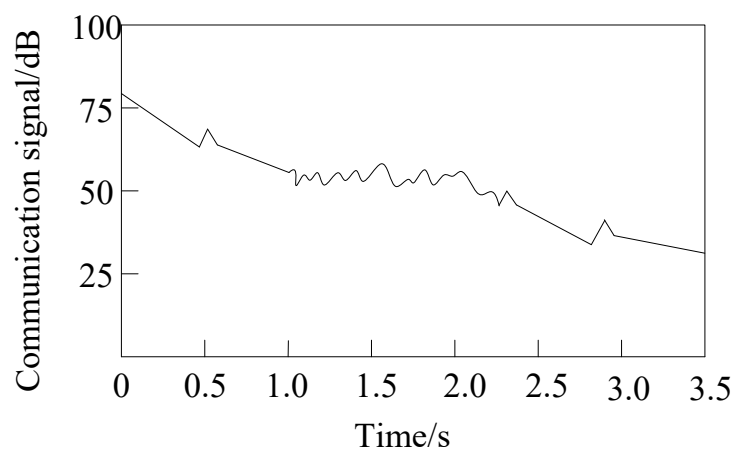

(c) topology feature 3 


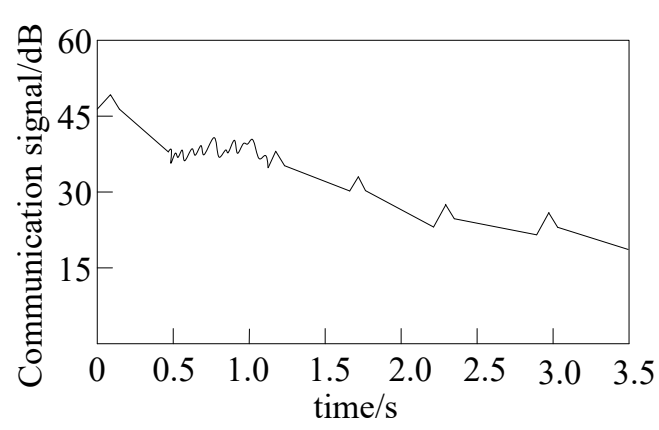

(d) topology feature 4

Fig. 5 topology feature

As can be seen from Fig. 5, the above four topological features are plotted according to the equation solution obtained by the specific solution method of solution flow a, B, C and D in Fig. 1. According to the above four topological features, the traditional application methods and the application methods based on topological features are compared and analyzed. The comparison results of network communication signals of the two methods are shown in Table III.

Table III. Comparison of network communication signals of the two methods

\begin{tabular}{cccc}
\hline Topology & $\begin{array}{c}\text { Traditional } \\
\text { application } \\
\text { method }\end{array}$ & $\begin{array}{c}\text { Application method } \\
\text { based on topology } \\
\text { features }\end{array}$ & $\begin{array}{c}\text { Standard } \\
\text { value }\end{array}$ \\
\hline 1 & $60 \mathrm{~dB}$ & $40 \mathrm{~dB}$ & $40 \mathrm{~dB}$ \\
2 & $53 \mathrm{~dB}$ & $45 \mathrm{~dB}$ & $45 \mathrm{~dB}$ \\
3 & $32 \mathrm{~dB}$ & $60 \mathrm{~dB}$ & $61 \mathrm{~dB}$ \\
4 & $36 \mathrm{~dB}$ & $41 \mathrm{~dB}$ & $41 \mathrm{~dB}$ \\
\hline
\end{tabular}

It can be seen from Table III that the difference between the communication signal based on topology features application method and the actual signal is small, while the difference between the network communication signal based on traditional application method and the actual signal is large.

In order to further verify the application effect of the application method based on topology features, it is necessary to compare the traditional method with the proposed method, and the results are shown in Table IV.

Table IV. Application effect of two methods

\begin{tabular}{ccc}
\hline Experiment/Time & $\begin{array}{c}\text { Traditional } \\
\text { method }\end{array}$ & $\begin{array}{c}\text { The proposed } \\
\text { method }\end{array}$ \\
\hline 1 & $45 \%$ & $98 \%$ \\
2 & $46 \%$ & $96 \%$ \\
3 & $52 \%$ & $96 \%$ \\
4 & $50 \%$ & $95 \%$ \\
5 & $49 \%$ & $95 \%$ \\
\hline
\end{tabular}

According to the above test contents, it can be concluded that the application effect of this method is more than $95 \%$, while the application effect of the traditional method is about $50 \%$. Compared with the traditional method, the application effect of this method is better, and the application effect of using this method to solve the nonlinear wave equation has been improved. The effectiveness of the method is verified.

\section{E. Discuss}

The existence and multiplicity of solutions of partial differential equations is not only an important field of modern science, but also an important part of the theory of partial differential equations. At present, the practical application effect of relevant research is poor. In this paper, the application of solving nonlinear wave equations based on topological characteristics is proposed. The main work of this paper is as follows:

(1) According to the characteristics of topology, the first-order integral method is used to solve the exact solution of nonlinear wave equation. The application effect of nonlinear wave equation is improved.

(2) According to the first integration method, the threshold range is set, and the solution process is designed according to the division theorem.

(3) Compared with random network and scale-free network, scale-free network has better completeness and higher network efficiency than random network in network characteristics. In order to express the structure of the network more clearly, the connectivity and circulation of the network can be evaluated by index, index and index.

The communication signal based on topology has little difference between the application method and the actual signal. The application effect of this method is more than $95 \%$. Compared with the traditional method, the application effect of this method is better. The application effect of using this method to solve the nonlinear wave equation has been improved. The application of topology to solving nonlinear wave equations is the research focus in this field. The research of this paper is to attract people's attention. I believe there will be more research results in the future.

\section{CONCLUSIONS}

In this paper, we mainly study the exact solutions of nonlinear wave equations, especially $(1+i)$ and $(2+1)$ dimensional nonlinear wave equations. Various local excitation modes and the dynamics of fractal and chaos are also analyzed. Here, the reduction idea of nonlinear equation and the separation variable theory of linear equation are mainly applied. First, the reduction idea of dealing with nonlinear inter problem and the multi linear separation variable method are studied and generalized, the deformation mapping theory is innovated, and some new results are obtained. Then, according to the reduction solution, mapping transformation solution and multilinear separated variable solution of the nonlinear system, the local excitation modes and the related nonlinear dynamic behaviors of $(1+1)$ and $(2+1)$ dimensional nonlinear systems are discussed. The results show that multilinear variable separation method, mapping transformation method and even symmetry reduction method can be unified. Through the research, we can understand the relationship between the ordered phenomena, chaos and 
Fractal in nature, which is helpful to deepen the understanding of nonlinear wave equation.

Generally speaking, for the high-dimensional system, in the low-dimensional function, the nonlinear wave should be satisfied. For different nonlinear equations, the general quantity corresponds to different physical quantity, which may be the field quantity of the system, the potential function and the square mode function of the amplitude. In any case, the general formula can be used to construct rich local excitation modes. In this paper, the general theory of hyperbolic function method for differential difference system is given, its extension form and its application in nonlinear discrete system. Then, the deformation mapping theory based on traveling wave reduction and the algebraic solution of nonlinear wave equation based on traveling wave reduction are given, and the approximate solution of higher-order nonlinear wave equation based on traveling wave under certain initial conditions is given.

Although some progress has been made in the innovation of basic theory, some new research results have been obtained in the application and promotion of methods. However, nonlinear science is still a young subject, and there is still a lot of work to be further studied. Several aspects are needed to make a brief outlook. By using the idea of direct reduction, a method of target reduction is proposed, which has been successfully applied to some nonlinear systems, and new exact solutions of these nonlinear systems are obtained. From the various patterns of the soliton structure, it can be seen that there is a certain difference between the target reduction method and the multilinear separation variable theory. However, the mapping transformation needs to be further improved. How to further extend the mapping transformation method to more nonlinear systems? So far, it has only been successfully applied to a few nonlinear equations. At the same time, in addition to Riccati equation (Group) mapping, Riccati equation generally refers to Riccati equation. Riccati equation is the simplest kind of nonlinear equation. There are also some very effective mapping equations (such as NKG equation and general elliptic equation) based on the mapping transformation method of traveling wave reduction. So, if NKG (one dimensional cubic nonlinearity) equation and general elliptic equation are taken as mapping equation, how about the study of mapping solution of the nonlinear system? This is an interesting and important topic.

\section{ACKNOWLEDGMENT}

Project supported by the National Natural Science Foundation of China (No. 11901320); Key Research Projects of Education University supported by Henan Provincial Department (No. 19A110028); Foundation and Frontiers Project supported by Henan Science and Technology Department (No. 162300410076).

\section{References}

[1] R. Carter, Y. E. Sánchez-Corrales, M. Hartley, V. A. Grieneisen, and A. F. M. Marée, "Pavement cells and the topology puzzle," Development, vol. 144, no. 23, pp. 4386-4397, 2017.

[2] C. P. Hoi, V. Ashvin, and W. Haruki, "Erratum: Symmetry-based indicators of band topology in the 230 space groups," Nature Communications, vol. 8, no. 1, pp. 50, 2017.

[3] S. Mattie, J. Riemer, J. G. Wideman, and H. M. McBride, "A new mitofusin topology places the redox-regulated C terminus in the mitochondrial intermembrane space," Journal of Cell Biology, vol. 217, no. 2, pp. 507-515, 2017.

[4] G. Xu, T. H. Kwok, and C. C. L. Wang, "Isogeometric computation reuse method for complex objects with topology-consistent volumetric parameterization," Computer-Aided Design, vol. 91, pp. 1-13, 2017.

[5] R. K. Mai, Y. Chen, Y. Li, Y. Y. Zhang, G. Z. Cao, and Z. $\mathrm{Y}$. He, "Inductive power transfer for massive electric bicycles charging based on hybrid topology switching with a single inverter," IEEE Transactions on Power Electronics, vol. 32, no. 8, pp. 5897-5906, 2017.

[6] F. Romanò, S. Albensoeder, and H. C. Kuhlmann, "Topology of three-dimensional steady cellular flow in a two-sided anti-parallel lid-driven cavity," Journal of Fluid Mechanics, vol. 826, pp. 302-334, 2017.

[7] P. Andrejic and J. Lekner, "Topology of phase and polarization singularities in focal regions," Journal of Optics, vol. 19, no. 10, pp. 105609, 2017.

[8] F. Gao, Y. Hu, G. Liu, and Y. Yang, "Experiment study on topological characteristics of sandstone coating by micro CT," Coatings, vol. 10, no. 12, pp. 1143, 2020.

[9] T. Chambrion and L. Thomann, "A topological obstruction to the controllability of nonlinear wave equations with bilinear control term," SIAM Journal on Control and Optimization, vol. 57, no. 4, pp. 2315-2327, 2019.

[10]A. I. Maimistov and E. I. Lyashko, "Spin angular momentum of a nonlinear surface wave at the interface between ordinary and topological insulators," Optics and Spectroscopy, vol. 126, no. 5, pp. 497-502, 2019.

[11] P. Bartesaghi, G. P. Clemente, and R. Grassi, "Clustering coefficients in weighted undirected multilayer networks," Papers, 2021.

[12]M. A. Midoun, X. Wang, and M. Z. Talhaoui, "A pyramidal community detection algorithm based on a generalization of the clustering coefficient," Journal of Ambient Intelligence and Humanized Computing, 2020.

[13] S. A. Amamra, K. Meghriche, A. Cherifi, and B. Francois, "Multilevel inverter topology for renewable energy grid integration," IEEE Transactions on Industrial Electronics, vol. 64, no. 11, pp. 8855-8866, 2017.

[14] Y. Jiao, Y. Wu, and S. Lu, "The role of crowdsourcing in product design: the moderating effect of user expertise and network connectivity," Technology in Society, vol. 64, no. 2, pp. 101496, 2021.

[15] A. Abdollahi and Z. Taheri, "Zero divisors of support size 3 in group algebras and trinomials divided by irreducible polynomials over gf(2)," Rendiconti del Seminario matematico della Università di Padova, vol. 145, 2021. 


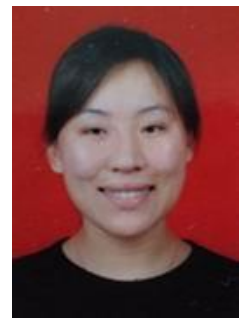

Liang Song, female, was born in September 1981. Her title is associate professor. She received a B.S degree in mathematics and applied mathematics from Wuhan University in 2005. She received the Master's degree in computer application technology from Huazhong University of Science and Technology in 2008. She is currently an associate professor in the School of Mathematics and Physics of Nanyang Institute of Technology. Her research interests are mainly in the areas of stability and filter. She has published twelve academic articles, and has participated in 6 scientific research projects.

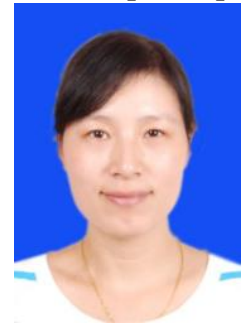

Guihua Li, female, was born in September 1981. Her title is lecturer. She received a B.S degree in mathematics and applied mathematics from Anyang Normal University in 2005. She is currently a lecturer in the School of Mathematics and Physics of Nanyang Institute of Technology. Her research interests are mainly in the areas of stability and filter. She has published six papers and participated in two research projects.

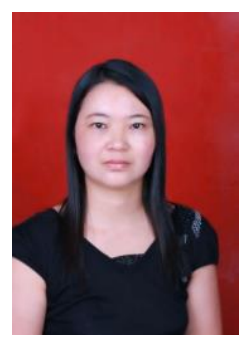

Shaodong Chen, female, was born in July 1968. Her title is associate professor. She received a B.S degree in mathematics and applied mathematics from Henan Normal University in 1991. She received the Master's degree in mathematics and applied mathematics from Henan Normal University in 2006. She works for the Nanyang Institute of Technology, Nanyang, China. Her research interest is mainly in the area of cybernetics. She has published more than twenty academic articles, and has participated in 5 scientific research projects.

\section{Author Contributions:}

Liang Song counted and analyzed the sample data. Guihua Li studied the nonlinear wave equation. Shaodong Chen analyzed the network topology. The experimental results show that the application of this method is $98 \%$, which is still effective for the hyperbolic development equation of the same type.

\section{Creative Commons Attribution License 4.0 (Attribution 4.0 International, CC BY 4.0)}

This article is published under the terms of the Creative Commons Attribution License 4.0 https://creativecommons.org/licenses/by/4.0/deed.en_US 